\title{
Healthcare professionals' perceptions of interacting with patients of South Asian origin attending early inflammatory arthritis clinics
}

\author{
Kanta Kumar ${ }^{1}$, Rebecca Stack $^{2}$, Ade Adebajo ${ }^{3}$, Jo Adams ${ }^{4}$
}

${ }^{1}$ Institute of Clinical Sciences, College of Medical and Dental Sciences, University of Birmingham, Birmingham, United Kingdom.

${ }^{2}$ Department of Psychology, Nottingham Trent University, Nottingham, United Kingdom,.

${ }^{3}$ Faculty of Medicine, Dentistry and Health, University of Sheffield, Sheffield, United Kingdom,

${ }^{4}$ School of Health Sciences, Faculty of Environment and Life Sciences, University of Southampton, Southampton, United Kingdom,.

Correspondence to: Kanta Kumar k.kumar@bham.ac.uk University of Birmingham, Birmingham, United Kingdom B15 2TT. Email: k.kumar@bham.ac.uk 


\begin{abstract}
Objective. To explore rheumatology healthcare professionals' (HCPs) perceptions of interacting with patients of South Asian origin attending early inflammatory arthritis (EIA) clinics.
\end{abstract}

Methods. We used face to face semi structured interviews, designed in partnership with clinician partner to interview ten HCPs involved in running of EIA clinics across seven centres in the UK. Data were recorded, transcribed by an independent company and were analysed using inductive thematic analysis.

Results. Three emerging themes were identified that characterised consulting experiences of HCPs. [1] Varied approaches were used in early inflammatory arthritis clinic, [2] Rheumatology HCP's challenges in managing and delivering information to patients of South Asian origin in early inflammatory arthritis clinic [3] Moving towards good practice: views on managing future patients of South Asian origin in early inflammatory arthritis clinics. Overall, HCPs found that they required additional skills to support the engagement and management for patients of South Asian origin living with inflammatory arthritis. HCPs felt that they were less effective in addressing self-management issues for this patient group and they found it difficult to determine adherence to medication. In such consultations, HCPs perceived their own limitation of inadequate training contributed towards poor consultations.

Conclusion. For the first time, our data demonstrates that the management of patients of South Asian origin in EIA clinics is under served. To address this, HCPs have identified training needs to improve knowledge and skills in engaging with and supporting patients of South Asian origin. These findings provide a good direction for future research.

Key words: rheumatology, health care professional interaction, early inflammatory arthritis

\title{
Rheumatology Key messages
}

HCPs express an active role in managing patients of South Asian origin but lack confidence.

Tailored training programmes may improve HCP's confidence in interacting with patients of South Asian origin.

The training needs to address both consultation skills and the establishment of culturally sensitive services. 


\section{Introduction}

Today, rheumatology departments around the world are seeking to promote a health care system that is patient centred in which the patients are actively involved in their treatment plan [1]. From a patient's perspective, the ideal health care setting that includes educational input about their disease together with help from a multidisciplinary team is favoured [2]. Because of this, the traditional "one size fits all" approach should be abandoned. Internationally, rheumatoid arthritis (RA) which is one of the most common forms of inflammatory disease has become an increasingly morbid and costly [3]. The disease significantly impacts on every aspect of patients' life particularly on work patterns which adds to the financial burden $[4,5]$. Effective disease management can be achieved by seeing the patient in early inflammatory arthritis (EIA) clinics. Early detection, initiation of treatments and positive patient engagement can control disease activity significantly [6]. However, fewer patients with RA achieve the control needed to avoid complications. Furthermore, minority ethnic populations are disproportionately at high risk of poorly controlled RA [7]. Lack of awareness about RA, delays in seeking medical help at the onset of symptoms and educational resources; as well as differences in knowledge and health beliefs about RA contribute to poor outcomes $[8,9]$.

There are marked differences between RA ethnic groups around the globe in terms of medication adherence and perceptions of RA being a curable disease [7, 9]. The world is increasingly becoming a global village and across chronic conditions, healthcare professionals (HCPs) see more culturally diverse patients than ever before with many different cultures, languages and traditions living together as multicultural societies [7, 10-13]. Like other parts of the world, UK also makes up $7.9 \%$ of minority ethnic population with Indians being the largest minority group followed by Pakistanis [13]. Consequently, together, South Asians make up an overwhelming majority of the ethnic minority population of the UK. Similar to other diseases $[10,11]$ in RA, cultural factors influence beliefs, behaviour, perceptions and emotions, all of which have been reported as important implications for health and health care $[7,9]$. Many challenges stem from the consultation process. Patients of South Asian origin with RA are reported to favour discussions about disease causation and treatments plans with a wider understanding that conceptualises their thinking [14]. Sometimes this approach may be viewed as 'difficult' by HCPs and may lead to negative judgements [14]. Psychological theories have widely captured patients' process of rationalisation about having long term conditions and treatment [15]. Despite these theories aiding HCPs' understanding on patients' perspectives, 
some rheumatology HCPs who deal with minority ethnic population are presented with challenges in engaging with patients of South Asian origin attending EIA clinics. These perspectives become important to explore since engaging with patients at an early stage provides a platform for long term relationship and optimising the best clinical outcomes [16]. In our recent study that explored South Asian patients' experiences of attending early inflammatory arthritis clinics highlighted that there was room for improving the care delivered to this population [17]. In that study patients made useful recommendations for future clinics. In order to implement the lessons learnt from the patients' perspectives, we wanted to widen our understanding of clinics' experiences. Currently, there is limited data that documents the experiences of rheumatology HCPs dealing with minority ethnic patients. This study explored rheumatology HCPs perception of interacting with patients of South Asian origin attending EIA clinics and aimed to understand the facilitators and barriers to effective clinical interaction among minority ethnic populations, with the consequent global lessons.

\section{Methods}

Theory is interchangeably used in qualitative research [18]. We began from the position that rheumatological conditions have public health dimensions[19]. The disease onset strongly shows patterns of cultural influences and social determinants of health $[19,20]$. To explore the interactions of HCPs with patients of South Asian origin our orientation of theory centred on thematic analysis. Thematic analysis is known as a method in its own right that is not bound to any theoretical framework complementing the pragmatist approach which we took in this study [21]. Pragmatism is guided by the researchers' desire to produce socially useful knowledge and takes a bottom up approach [21]. An in-depth, qualitative interview study involving ten rheumatology healthcare professionals across seven centres in the UK including a mixture of consultants, registrars and specialist nurses was conducted. We used thematic principles as it allows the researcher to collect data for the purpose of generating a framework of understanding the HCPs experiences $[18,22]$. The reporting of this study falls in line with consolidating criteria for reporting qualitative research [23]. Using purposive sampling, we recruited HCPs from White British, Afro-Caribbean and South Asian backgrounds, those who spoke South Asian languages and those who did not and a mixture of gender (Table 1). The recruitment of HCPs took place from academic linked hospitals and general hospitals. This enabled us to explore a broad range of HCPs' experiences serving South Asian populations in EIA clinics. A semi-structured interview schedule designed and developed with a clinician partner was used to conduct ten interviews with rheumatology HCPs who were involved in 
running of EIA clinics (Table 2). Ethics approval was granted by the South West-Frenchay Research Ethics Committee (Reference 234815). Rheumatology HCPs were contacted by email via regional rheumatology mailing list and were invited to take part in qualitative interviews lasting approximately 45 minutes on their experiences of interacting with patients from South Asian background in EIA clinics. Those who expressed an interest were interviewed (more details under result section). Written informed consent was obtained from each HCP prior to interview. Interviews were conducted by $\mathrm{KK}$ an experienced researcher with rheumatology expertise. KK is also of Indian origin. Eight interviews were conducted face-toface and took place in the hospital rheumatology department where the participant practiced; and 2 were conducted over the telephone. Interviews were audio-recorded, anonymised and transcribed verbatim by an independent transcribing company.

\section{Data analysis}

Since our study was not based on grounded or other phenomenology framework, data were analysed using a form of thematic analysis. Analysis began shortly after initial data collection. An iterative coding procedure in accordance with principles of inductive thematic analysis was used [22]. An analysis approach appropriate to applied health services research, enabling investigation of issues while simultaneously allowing for identification of newly emergent ideas in the data was used [22]. For all interviews the progressing analysis informed subsequent data collection. At the end of each interview a summary of findings was discussed with HCPs for agreement. Interviews continued until main data categories were saturated and no new insights were apparent. The team included diverse expertise: a rheumatologist (AA), health psychologist (RS), research partner (EP), and health literacy expert (JA) and all contributed different expertise to data analysis.

Rigour was achieved through a process of reflexivity and by documenting all analytic decisions, leaving an audit trail. The first author (KK) systematically analysed all the transcripts to establish themes grounded in the data. In this process, transcripts were read and reread line by line. Clinician research partner (EP) independently coded three transcripts to develop reliable and inclusive themes informed by multiple perspectives. The triangulation process and discussion of the coding framework took place between researchers (KK, AA, RS, EP and JA) who compared codes to solve any differences. This process allowed researchers to validate trustworthiness of the data. All transcripts were checked for the emerging new codes identifying data saturation. The team further read and analysed random selection to confirm 
interpretation of codes. Codes were grouped into categories discussed and revised by the team and then were grouped into themes. Coding categories that lacked concordance were discussed and absorbed into the coding framework. The core themes extracted and presented here focus on HCPs' perceptions of interaction with patients from South Asian background in EIA clinics. Initially, 182 codes were identified, which were then grouped into 35 categories and finally combined into three overarching themes. The final report was sent to participants who did not make any changes.

\section{Results}

Ten rheumatology clinicians (six rheumatologists, four nurses), from seven academic linked and National Health Service (NHS) hospitals across the UK took part. In total 18 were approached to take part in the study. However, due to work commitments eight HCPs were unable to fix a time for interview. The declined population included five male and three females. Of the ten who took part, there were seven women and three men, ranging in age from 29 to 62 years (mean 45.6) and qualified from 8 to 35 (mean 20.3) years. Suitable quotes were selected to illustrate the findings. These are presented in Table 3. The three predominant themes enhanced our understanding of HCPs' perceptions of interacting with patients from South Asians background in EIA clinic. See Figure 1 for a thematic diagram of HCPs' perceptions of interacting with patients of South Asian origin in the EIA clinic and supporting the management of RA.

\section{Varied approaches used in early inflammatory arthritis clinic}

Rheumatology HCPs in the study described their roles. The doctors viewed their aim of consultation being focused primarily on diagnosis, assessing physical symptoms and function (Q1). The clinical nurse specialist (CNS) described their role more broadly where they included a focus on educating patients and providing them with information on lifestyle changes (Q2). The doctors were familiar with a model of care that emphasised a holistic mind and body interaction and recognised RA as a complex disease with a range of psychological, social and physical sequelae but questioned if needs of patients were addressed adequately (Q3). The HCPs acknowledged the patient perceived burden of having RA would impact patients from South Asian background in many different ways (Q4). Some doctors reported to use goal setting techniques with their patients however; this approach was used only amongst a few doctors from minority ethnic background (Q5). Doctors from White background accounts also described less sophisticated management approaches that weren't embedded within 
behavioural theoretical models (Q6). Although the CNSs described a broader role to play in EIA clinics they too were less likely to use strategies that motivate patients at an early stage in the minority ethnic background patients (Q7). Both doctors and CNSs tended to use standard leaflets to help support their discussions during consultations. There was recognition that the leaflets presented a limitation for non-English speaking patients (Q8). Only one centre used visual representation to communicate disease related information. The HCP at this centre expressed the view that the visual representation material helped engagement with the problem and need for treatment. Particularly, for patients with lower literacy level (Q9).

Within their varied approaches used to engage patients from South Asian background there was lack of knowledge about resources available that could assist HCPs in educating patients of South Asian origin (Q10). Only a few centres were aware about National Rheumatoid Arthritis Society (NRAS) Apni Jung webpage (our fight against rheumatism) (www.nras.org.uk/apnijung). The reason for this was cited as lack of time to sign post and for some lack of knowledge about this project. HCPs recognised that RA could be a demanding condition with impact on patients' emotional wellbeing and quality of life however they were less likely to undertake structured behavioural approaches. HCPs concurred on the perceived lack of shared management of RA in patients of South Asian origin and that the condition was not subject to the same level care as other long term diseases such as diabetes. Specifically, patients' need for information, decisions to take medication, support for self-care and coping was problematic (Q11). Systems for adequately supporting these needs were perceived as lacking in EIA clinics.

Example of HCPs quotes:

Q4. I do feel it's really tricky to engage with patients from South Asian backgrounds in the inflammatory arthritis clinics. Particularly the women I don't have the same feeling for the men at all but the women can be very different and do perceive RA to be burdening more than others. [White British, consultant, Female]

Q6. I don't tend to do the goal setting as I don't know much about the culture really. I tend to ask how they are feeling and that sometimes gives me enough to know if the condition is controlled or not. I don't use a framework and perhaps I should because we do use a framework for our young adolescents and that works well. This is good point why don't we use this for South Asians. [White British, consultant, Male] 


\section{Experience of managing and delivering information to patients from South Asian background in early inflammatory arthritis clinic: rheumatology healthcare professionals' challenges}

HCPs considered consultation for wide spread pain to be higher among patients of South Asian origin compared to the indigenous UK population. They reported that it was not common for patients of South Asian origin to consult with symptoms but present these as new at every visit, possibly in the hope of obtaining alternative medication or cure (Q12). The HCPs were familiar with the concept of illness belief models that some patients of South Asian origin hold and they recognised their own limitations in informing these with their patients during clinic consultation. HCPs reflected on their experiences and noted South Asians take longer to make decisions about their treatments therefore, require more time and sometimes extra clinical visits (Q13). The HCPs expressed despite their effort in supporting self-management some patients of South Asian origin validated information with families and did not always attend the multidisciplinary team appointments. An example of this was physiotherapy (Q14). HCPs expressed difficulties associated with engaging some patients and often noted that their approach and methods were not effective in engaging patients of South Asian origin attending physiotherapy appointments. Only one centre included physiotherapy treatment as being part of the consultation supporting patients to understand the role of the multidisciplinary team and purpose of involving different HCPs in their care (Q15).

HCPs suggested providing information in English to non- English speaking patients of South Asian origin on RA and treatments were not often seen as useful. Moreover, HCP, felt that non-English patients of South Asian origin did not actively seek information about their RA and management (Q16) and that HCPs should provide some additional support to facilitate this. Some consultants reported their experience of hierarchy amongst first generation patients where patients viewed a doctors' opinion to be more valued than other HCPs (Q17). HCPs who were from the same ethnic background as the patient found communicating in the patient's native language helped build better rapport and enabled such approaches as goal setting to be implemented much more effectively (Q18). In contrast, HCPs who were from White origin backgrounds expressed challenges in engaging with some Asian patients particularly those who were non-English speaking (Q19). Due to cultural barriers some doctors from a White origin background acknowledged difficulties managing patients of South Asian origin. This mirrored the accounts of doctors who believed there was little they could do to help with RA management and were consequently reluctant to consult fully with patients (Q20). The manner 
in which patients of South Asian origin present and express their concerns was perceived to be culturally influential during the consultation process. Many HCPs reported that patients of South Asian origin present more frequently (Q21). HCPs described circumstances, where clinic presentations were florid, posing challenges and frustration for the HCPs to treat and manage patients (Q21). The HCPs found second and third generation South Asians presented differently from non-acculturated South Asians and were similar to patients from White British background (Q22).

The difficulties associated with managing patients of South Asian origin living with RA pose personal challenges for HCPs causing some HCPs to question their training and value to the community (Q23). Adherence to medication was also noted and difficult to determine during consultation. HCPs reported feelings of their own limitations and lack of knowledge and skills when patients repeatedly failed to adhere to previously prescribed medication and it was acknowledged that a focus on disease cure was prioritised by patients (Q24). HCPs reported that building a strong doctor-patient relationship was important however, this was perceived to take a long time with patients of South Asian origin for HCPs who were from White origin background.

Examples of HCPs quotes:

Q12. I know some of them want the cure and maybe that's why they don't like to take all these medications because they know it will never go away. Some of them do have more pain and it is repeated in consultations. [Indian, consultant, Male]

Q19. I grew up in an environment where there was very little ethnic populations. I now work in here and I don't really know what the expectations are from disease and sometimes it is hard to unpick these things. [White British, Registrar, Female]

\section{Moving towards good practice: views on managing future patients from South Asian background in early inflammatory arthritis clinics}

HCPs in this study discussed the concept of a collaborative relationship between clinician and patient and noted this to be the cornerstone of more effective patient engagement. However, due to their own lack of awareness about different cultural values and how to tackle some health beliefs held by minority ethnic populations; meant they lost the opportunity to explore these and were unlikely to apply goal setting techniques during consultation (Q25). A few doctors suggested having departmental champions who could act as ambassadors for updating on 
health diversity agendas as some were not fully aware of updated research on ethnicity in rheumatology practice (Q26).

Most HCPs from White origin background accounts indicated self-reported low levels of expertise and confidence in the management of patients from minority ethnic backgrounds living with RA, blaming lack of training opportunities (Q27). This appeared more apparent for those HCPs who might have trained in non-ethnic diverse environments but now working in hospitals serving large amount of minority ethnic population (Q28). In contrast, HCPs of South Asian origin felt they were at an advantage of communicating with patients of South Asian origin.

During the interview many White British origin HCPs reflected and recognised the needs towards developing cultural specific skills that could better assist them in engaging minority ethnic population in consultation. All HCPs expressed having skills to apply theory underpinning illness health beliefs and self-management would be useful to strengthen their' confidence in managing minority ethnic population better (Q29). HCPs stated that it would be helpful in trying new approaches, which might offer more to both patient and HCPs given the time constrains to see patients. A concern experienced by many HCPs was in relation to using new skills competently in minority ethnic patients, and how to respond if complex psychological difficulties emerged in a consultation (Q30). A clear sense emerged that formal training would be the start of a learning process for managing patients from minority ethnic backgrounds. HCPs described how they could use theoretically driven strategies to support minority ethnic population and enhance better shared care decision making in clinical practice after training (Q31, 32, 33 \& 34).

Examples of HCP quotes:

Q31. I think we should have a programme for professionals as cultures and beliefs are very different and trying to make sense of these can be a job. We only need to look at other specialities like cancer they do... [Afro-Caribbean, registrar, Female]

Q28. I think that's something we can learn in these training sessions coming from White 
backgrounds don't always allow the discussions. I am typical middle class person who has little knowledge about different cultures the things I have learnt are what I have picked up along the way. I think learning more about these models might be more useful in that training for people like me and being verified by people maybe like you who know the research would be a great resource. [White British, consultant, Female]

\section{Discussion}

This is the first study to present the perspectives of rheumatology HCPs' perceptions of interacting with patients of South Asian origin attending EIA clinics. We have documented different approaches used by HCPs in engaging patients from minority ethnic backgrounds but only a very few consultations were based on any underpinning behavioural change theory. There were many challenges displayed by HCPs and signposting to specific resources that could have allowed patients of South Asian origin to better engagement were not always optimised. Educational materials currently available to non-English speaking patients of South Asian origin were also presented as challenges by HCPs.

We have found two key directions from HCPs that affirm future steps. Firstly, that all HCPs may require a better understanding of implementing health related theory models and skills such as motivational interviewing into their consultation when engaging with patients of South Asian origin. In our study, HCPs identified the need for training and reflected that theory models could be better used to guide consultations. Theory based interventions with advanced skills have been tested and held high in improving patient-clinician relationship [24]. Allowing opportunity to generate an improved engagement and increase the likelihood of wide adoption of self-management by patients [25]. In our study where experiences of South Asians attending the early inflammatory arthritis clinics were explored reported a massive impact of RA on patients' psychological status [17]. The psychological burden and tension was reported to increase due to lack of effective interaction with HCPs to manage RA. The results of HCPs experiences of interacting with patients of South Asian origin affirm the reasons for lack of interactions in clinics. Our data suggest an opportunity to help embed skills in clinical practice, and develop advanced techniques to move forward. Transfer of training theory proposes that higher levels of shared care decision making might increase the likelihood of the successful outcomes [24]. This was found in research into brief cognitive-behavioural training for clinicians working in cancer [26], where clinical consultation was based on necessity and needs 
of patients HCPs trained to address concerns effectively, maintain skills and build confidence. In our study, HCPs in one centre identified the creation of advanced technique and employed different mode of communication where by diagrammatic and use of showing pictures of joints during ultrasound consultation, was found particularly useful for non-English speaking patients. That centre used this approach to communicate disease related information in patients with lower literacy levels and perhaps less acculturated in the western systems. This extends our understanding that going beyond traditional consultations styles may reap meaningful rewards. For example, pictorial or showing visualise type of material can be helpful to communicate disease related information [27]. Surprisingly, the use of visualisation during consultation was only used in one centre yet support from previous studies have shown this method of engagement to help patients' reduce their treatment related concerns that lead to non-adherent behaviours particularly the case among patients of South Asian origin living with RA [7]. Moreover, other specialities have shown visualisation when used within SelfRegulation Theory $[28,29]$ assist patients in rationalising their disease process in understand long term need for treatment [30, 31]. Particularly helpful for engaging minority ethnic population who have been known to seek a cure of the disease and often lack motivation to adhere to treatment [32] moreover, were reported to have frequent hospitals visits in our study.

Secondly, HCPs who were not from minority ethnic background strongly recommended advanced cultural competency training for rheumatology practitioners. Our findings resonate strongly with those of previous researchers [33], who have shown that HCPs consulting with minority ethnic populations are not always equipped with appropriate training to address the cultural sensitive issues and how these drive clinical decisions [34, 35]. Cultural competence training in speciality like diabetes has been shown to improve patient outcomes $[10,36]$. A systematic review of 34 studies by Beach et al [37] found that training clinicians improved knowledge in 17 of 19 studies skills and attitudes in 21 of 25 studies. Similarly, another systematic review [38] found five studies showed that cultural competence training of HCPs resulted in an improved patient satisfaction and better understanding of treatment plan [39]. Others noted an improvement in clinical variables in diabetes [39]. This was echoed by another systematic review [40] where 13 studies of HCPs and diabetic patients concluded that cultural competence training increased clinicians' knowledge about and awareness of dealing with culturally diverse patients and cultural sensitivity. Moreover, cultural awareness has been highlighted in areas like general practitioners (GPs) [41]. 
Our study has limitations; we recruited a relatively small sample size which may not have enabled us to reach data saturation. However, we were able to explore a broad overview of the rheumatology HCPs' perspectives of interacting with South Asian patients attending EIA clinics across seven centres in the UK. Alternative methodology such as the use of focus groups could have enabled a more in depth analysis of each clinic which may have offered useful insights for patient journeys and experiences. Having conducted our interviews face to face and over the telephone could have introduced some unintentional bias. Nevertheless, we elucidated the views of HCPs and capturing their experiences on what would help conduct effective consultations in EIA clinics across the UK was achieved.

\section{Conclusions}

It is clear that rheumatology HCPs particularly those from non-South Asian backgrounds believe they need advanced understanding of cultural skills training and currently find consultations difficult when dealing with minority ethnic patients. Moreover, all HCPs may require better understanding of theory related health models to enhance consultations. The training needs to address both consultation skills and the establishment of culturally sensitive services. These findings provide a good direction for future research. Our study provides an exemplar that can be utilised internationally for all rheumatology departments that treat patients from a variety of ethnic backgrounds. The issues identified here should provide an example for all health care professionals working to develop culturally competent staff and deliver culturally appropriate services to all patients.

\section{References}

1.Zangi HA, Ndosi M, Adams J, Andersen L, Bode C, Bostrom C, et al. EULAR recommendations for patient education for people with inflammatory arthritis. Ann Rheum Dis. 2015;74(6):954-62.

2.Dures E, Almeida C, Caesley J, Peterson A, Ambler N, Morris M, et al. Patient preferences for psychological support in inflammatory arthritis: a multicentre survey. Ann Rheum Dis. 2016;75(1):142-7.

3.Smolen JS, Landewe R, Bijlsma J, Burmester G, Chatzidionysiou K, Dougados M, et al. EULAR recommendations for the management of rheumatoid arthritis with synthetic and biological disease-modifying antirheumatic drugs: 2016 update. Ann Rheum Dis. 2017;76(6):960-77. 
4.Young A, Dixey J, Kulinskaya E, Cox N, Davies P, Devlin J, et al. Which patients stop working because of rheumatoid arthritis? Results of five years' follow up in 732 patients from the Early RA Study (ERAS). Ann Rheum Dis. 2002;61(4):335-40.

5.Looper KJ, Mustafa SS, Zelkowitz P, Purden M, Baron M. Work instability and financial loss in early inflammatory arthritis. Int J Rheum Dis. 2012;15(6):546-53.

6.Smolen JS, Landewe R, Breedveld FC, Buch M, Burmester G, Dougados M, et al. EULAR recommendations for the management of rheumatoid arthritis with synthetic and biological disease-modifying antirheumatic drugs: 2013 update. Ann Rheum Dis. 2014;73(3):492-509.

7.Kumar K, Raza K, Nightingale P, Horne R, Chapman S, Greenfield S, et al. Determinants of adherence to disease modifying anti-rheumatic drugs in White British and South Asian patients with rheumatoid arthritis: a cross sectional study. BMC Musculoskelet Disord. 2015;16(1):396.

8.Kumar K, Daley E, Khattak F, Buckley CD, Raza K. The influence of ethnicity on the extent of, and reasons underlying, delay in general practitioner consultation in patients with RA. Rheumatology (Oxford). 2010;49(5):1005-12.

9.Kumar K, Gordon C, Toescu V, Buckley CD, Horn R, Nightingale PG, et al. Beliefs about medicines in patients with RA and SLE: a comparison between patients of South Asian and White British origin. Rheumatology. 2008;47((5):):690-7.

10.Greenhalgh T, Clinch M, Afsar N, Choudhury Y, Sudra R, Campbell-Richards D, et al. Socio-cultural influences on the behaviour of South Asian women with diabetes in pregnancy: qualitative study using a multi-level theoretical approach. BMC Med. 2015;13:120.

11.Douglas A, Bhopal RS, Bhopal R, Forbes JF, Gill JM, Lawton J, et al. Recruiting South Asians to a lifestyle intervention trial: experiences and lessons from PODOSA (Prevention of Diabetes \& Obesity in South Asians). Trials. 2011;12:220.

12. Worth A, Irshad T, Bhopal R, Brown D, Lawton J, Grant E, et al. Vulnerability and access to care for South Asian Sikh and Muslim patients with life limiting illness in Scotland: prospective longitudinal qualitative study. BMJ. 2009;338:b183.

13.Bhopal R. Medicine and public health in a multiethnic world. J Public Health (Oxf). 2009;31(3):315-21.

14.Kumar K, Raizada SR, Mallen CD, Stack RJ. UK-South Asian patients' experiences of and satisfaction toward receiving information about biologics in rheumatoid arthritis. Patient Prefer Adherence. 2018;12:489-97.

15.Horne R, Chapman SC, Parham R, Freemantle N, Forbes A, Cooper V. Understanding Patients' Adherence-Related Beliefs about Medicines Prescribed for Long-Term Conditions: A Meta-Analytic Review of the Necessity-Concerns Framework. PLoS One. 2013;8(12):e80633. 
16.Raza K, Buckley CE, Salmon M, Buckley CD. Treating very early rheumatoid arthritis. Best Pract Res Clin Rheumatol. 2006;20(5):849-63.

17.Kumar K RJ, Stack RJ, Adebajo A, Adams J. Experiences of South Asian patients in early inflammatory arthritis clinic: a qualitative interview study. Rheumatology Advances in Practice. 2019; Volume 3, (Issue 2,) doi.org/10.1093/rap/rkz017

18.Bradley EH, Curry LA, Devers KJ. Qualitative data analysis for health services research: developing taxonomy, themes, and theory. Health Serv Res. 2007;42(4):1758-72.

19.Kumar K, Klocke R. Ethnicity in rheumatic disease. Clin Med (Lond). 2010;10(4):370-2.

20.Samanta A, Samanta J, Johnson M, Brooks N. Rheumatoid arthritis in minority ethnic groups: patterns of disease, clinical and sociocultural features among British South Asians. Diversity in Health and Social Care. 2005;2:99-118.

21.Meyer S WP. How to use social theory within and throughout qualitative research in healthcare contexts Sociol Compass. 2014;8(5):525 -39.

22.Dixon-Woods M, Agarwal S, Jones D, Young B, Sutton A. Synthesising qualitative and quantitative evidence: a review of possible methods. J Health Serv Res Policy. 2005;10(1):4553.

23.Tong A, Sainsbury P, Craig J. Consolidated criteria for reporting qualitative research (COREQ): a 32-item checklist for interviews and focus groups. Int $\mathrm{J}$ Qual Health Care. 2007;19(6):349-57.

24.Phillips LA, Leventhal H, Leventhal EA. Physicians' communication of the common-sense self-regulation model results in greater reported adherence than physicians' use of interpersonal skills. Br J Health Psychol. 2012;17(2):244-57.

25.Dures E, Hewlett S, Ambler N, Jenkins R, Clarke J, Gooberman-Hill R. Rheumatology clinicians' experiences of brief training and implementation of skills to support patient selfmanagement. BMC Musculoskelet Disord. 2014;15:108.

26. Melton L. Brief Introduction to Cognitive Behavioral Therapy for the Advanced Practitioner in Oncology. J Adv Pract Oncol. 2017;8(2):188-93.

27.Joplin SK, van der Zwan R, Bagga H, Joshua F, Wong PK. Pilot study assessing the novel use of musculoskeletal ultrasound in patients with rheumatoid arthritis to improve patient attitudes and adherence to medication. Int J Rheum Dis. 2014.

28. Shahab L, Hall S, Marteau T. Showing smokers with vascular disease images of their arteries to motivate cessation: a pilot study. Br J Health Psychol. 2007;12(Pt 2):275-83. 
29. Bovet P, Perret F, Cornuz J, Quilindo J, Paccaud F. Improved smoking cessation in smokers given ultrasound photographs of their own atherosclerotic plaques. Prev Med. 2002;34(2):21520 .

30.El MY, El GM, Palmer D. Assessment of the utility of visual feedback in the treatment of early rheumatoid arthritis patients: a pilot study. Rheumatol Int. 2012;32(10):3061-8.

31.Tadmor OP, Zlotogorski Z, Galron-Duniec M, Rabinowitz R, Neuman M, Beller U, et al. The effect of feedback on anxiety levels during ultrasound scanning for ovarian cancer. Ultrasound Obstet Gynecol. 1995;6(2):135-9.

32.Kumar K, Raza K, Gill P, Greenfield S. The impact of using musculoskeletal ultrasound imaging and other influencing factors on medication adherence in patients with rheumatoid arthritis: a qualitative study. Patient Prefer Adherence. 2016;10:1091-100.

33.Sopoaga F, Zaharic T, Kokaua J, Covello S. Training a medical workforce to meet the needs of diverse minority communities. BMC Med Educ. 2017;17(1):19.

34.Paternotte E, van Dulmen S, van der Lee N, Scherpbier AJ, Scheele F. Factors influencing intercultural doctor-patient communication: a realist review. Patient Educ Couns. 2015;98(4):420-45.

35.Aggarwal NK, Pieh MC, Dixon L, Guarnaccia P, Alegria M, Lewis-Fernandez R. Clinician descriptions of communication strategies to improve treatment engagement by racial/ethnic minorities in mental health services: A systematic review. Patient Educ Couns. 2016;99(2):198-209.

36.Greenhalgh T, Campbell-Richards D, Vijayaraghavan S, Collard A, Malik F, Griffin M, et al. New models of self-management education for minority ethnic groups: pilot randomized trial of a story-sharing intervention. J Health Serv Res Policy. 2011;16(1):28-36.

37.Beach MC, Price EG, Gary TL, Robinson KA, Gozu A, Palacio A, et al. Cultural competence: a systematic review of health care provider educational interventions. Med Care. 2005;43(4):356-73.

38.Chipps JA, Simpson B, Brysiewicz P. The effectiveness of cultural-competence training for health professionals in community-based rehabilitation: a systematic review of literature. Worldviews Evid Based Nurs. 2008;5(2):85-94.

39. Sequist TD, Fitzmaurice GM, Marshall R, Shaykevich S, Marston A, Safran DG, et al. Cultural competency training and performance reports to improve diabetes care for black patients: a cluster randomized, controlled trial. Ann Intern Med. 2010;152(1):40-6.

40.Renzaho AM, Romios P, Crock C, Sonderlund AL. The effectiveness of cultural competence programs in ethnic minority patient-centered health care--a systematic review of the literature. Int J Qual Health Care. 2013;25(3):261-9. 
41.Ali N, Atkin K, Neal R. The role of culture in the general practice consultation process. Ethn Health. 2006;11(4):389-408.

\section{Figure legend}

Figure 1. Thematic diagram of healthcare professionals' experiences of interacting with patients of South Asian origin

Acknowledgement: we thank all healthcare professionals for taking part in the study. We thank our clinician research partner (Emma Powell) for assisting us with study design, development of interview guide and data analysis.

Funding: This grant was funded by British Society for Rheumatology [grant number 17-1420]. The funder had no role in study design, data collection and analysis, decision to publish, or preparation of the manuscript.

Disclosure statement: the authors have declared no conflict of interest.

Table 1: Demographic data of healthcare professionals interviewed

\begin{tabular}{|l|l|l|}
\hline Characteristics & Female & Male \\
\hline Age & 3 & \\
$25-40$ & 2 & 3 \\
$41-55$ & & \\
Self-identified ethnicity & 3 & 1 \\
White British & 2 & 0 \\
Afro-Caribbean & 1 & 1 \\
Indian & 1 & 1 \\
British Indian & & 2 \\
Role type & 4 & - \\
Rheumatology Doctors & 4 & \\
Clinical Nurse Specialist & & \\
\end{tabular}


Table 2: topics discussed in interviews

\section{Topics}

1. Current role in early inflammatory arthritis clinic.

2. Experience of giving information to patients from South Asian background in the early inflammatory arthritis clinics

Examples of questions:

How do you tend to deliver disease related information to patients of South Asian origin?

Do you think the way this is done currently facilitates medication adherence and patient motivation?

Do you assess patients' health or illness beliefs?

What information do you use to assess those?

3. Views on future developing and improvements in early inflammatory arthritis clinics. Examples of questions:

How culturally sensitive do you think these approaches are?

What can aid a better patient engagement in your view? What resources might help? 
https://mc.manuscriptcentral.com/rheumap 
Table 3 Healthcare professionals' quotes

\begin{abstract}
Quotes relating to: Varied approaches used in early inflammatory arthritis clinic
Q1. My role is to diagnose patients and recommend treatment. If they need extra encouragement then the nurses normally do that. [White British, consultant, Female]

Q2. I mainly see patients to educate them and monitor their drugs. Inform them about disease process and DMARD therapy and how we will aim to treat. I educate about the lifestyle changes patients need to make. [British Indian, CNS, Female]
\end{abstract}

Q3. I would say certainly there are complex issues to deal with and one has to take a holistic approach but who is the person best to do this? That's the question. [White British, Registrar, Female]

Q4. I do feel it's really tricky to engage with patients from South Asian background in the inflammatory arthritis clinics. Particularly the women I don't have the same feeling for the men at all but the women can be very different and do perceive RA to be burdening more than others. [White British, consultant, Female]

Q5. I tend to start off by saying what is it that you would like to achieve for some it might be just making chapatti is important for some it might be looking after the grandchildren. But at least this gives me an idea of what the goal is and I like to make it person to them. I think if we can find someone who works with a goal then that becomes easier to work with. [Indian, consultant, Female]

Q6. I don't tend to do the goal setting as I don't know much about the culture really. I tend to ask how they are feeling and that sometimes gives me enough to know if the condition is controlled or not. I don't use a framework and perhaps I should because we do use a framework for our young adolescents and that works well. This is good point why don't we use this for South Asians. [White British, consultant, Male]

Q7. There isn't a formal tool that we use anything like that it is more what patients express. We normally would like to discuss this with patients. [British Indian, CNS, Female]

Q8. The leaflets are not good at getting across the harm and benefit balance. I think that most Leaflets have been developed with White patients and so therefore might not have the input of South Asian or ethnic groups. I suspect that section in the information leaflets is missing. Leaflets they don't have a reflections from Black minority ethnic groups views on board. I know they revise these sometime may be every year or so but they are mainly done by White patients. So there you go how we can make things more cultural sensitive. [Afro-Caribbean, Registrar Female]

Q9. You see what we found is that showing patients their problem with joints through an Ultrasound helps patients to really get to grips with the condition. May be this is more than words can describe. Pictures or making it real with scan might convince this population. [White British, CNS, Female]

Q10. I think we don't get to find this (referring to Apni Jung project) information there needs to be more education about these resources. We don't have enough time in clinic. [White British, consultant, Male] 
Q11. I do get concerned about the level of support that there is for South Asian patients really. I mean if we look at the diabetic care there is vast amount of information and we in rheumatology have very little. On reflection, we can't blame the patients disengagement. We have leaflets which are all in English. We don't have any cultural understanding other than our own experiences. It does need improving. We are producing more and more medications but patients or certain groups are not willing to take the general things so how will they take the other advanced ones. [White British, consultant, Female]

Quotes relating to: Experience of managing and delivering information to patients from South Asian background in early inflammatory arthritis clinic: rheumatology healthcare professionals' challenges

Q12. I know some of them want the cure and maybe that's why they don't like to take all these medications because they know it will never go away. Some of them do have more pain and it is repeated in consultations. [Indian, consultant, Male]

Q13. I think we only learn from our clinical experience about some problems but not sure how to deal with them. I do note there are very embedded health beliefs and when patients present in clinic we are not sure how to deal with those and in fact to address them. Clinics take longer and extra time. [White British, consultant, Female]

Q14. I feel there is a framework to discuss with family then it gets decided what will happens. It is really sad that they don't view the importance of exercising joints. My patients just don't attend the physio appointments. I know this because there is a letter dedicated to say the person never turned up so options are limited. [Afro-Caribbean, consultant, Female]

Q15. We include the physiotherapy in our consultations. We have found that in this way our patients will understand the holistic approach and who is who in the team. For South Asian patients we note they are more likely to attend the appointments for physio if they knew their role in self-managing. [British Indian, consultant, Female]

Q16. Sometimes the information is not taken seriously and this is more of a problem with the

non-English speaking. Then my view is well if you are going to listen to what I have to say then you should at least seek all this from somewhere. So I am aware that this goes on a lot at

the beginning of this journey and it is long time for having the disease. [White British, CNS, Female]

Q17. I would say certainly more hierarchy in the ethnic groups they do view the doctor to be a lot more important. So if our nurses see a patient and they are not very convinced then they will call me in to discuss this further with the patient. But when I doctor come in there might be more serious to take the advice. So occasionally we are asked to add that extra visits.

[Indian, consultant, Female]

Q18. To be fair I can understand the patients perceptive and try to get them engage may be better than my White colleagues. I speak Punjabi and Hindi so sometimes speaking in own mother tongue can help as well. It makes that connection with patients they are open to more 
about their plans for example like I said if they are not taking $15 \mathrm{mgs}$ of methotrexate then they will tell me what they are doing like taking the other stuff from India or something. [Indian, consultant, Female]

Q19. I grew up in an environment where there was very little ethnic populations. I now work in here and I don't really know what the expectations are from disease and sometimes it is hard to unpick these things. [White British, Registrar, Female]

Q20. I think as health professionals there is only so much you can do to help others. I mean South Asian patients particularly non-English speaking decide not to take on what we say therefore, there not much I can do about that. [White British, consultant, Male]

Q21. There is a lot of weighing up and down going on. There is also a lot of discussion with family and this takes some time for patients to decide about medication. Sometimes you never know what the patient or family want so just have to go with the flow. Sometimes this causes more discussion and more appointments. I find sometimes it is very challenging and frustrating to know how to move forward. [White British, consultant, Female]

Q22. I find a big difference in generations like the more acculturated ones will understand our

view point but first generation are not always on board. [White British registrar, Female]

Q23. I think to a certain extent we do lack knowledge about how to deal with ethnic minority populations. I mean do we even know how to address some of the things? I bet some us don't really get to the bottom of a consultation. I do think we need to improve this if we are to move forward. [White British, consultant, Female]

Q24. There might be the fact that people are not happy to take long term medication I can normally tell that from their body language. We can only tell them what we know and give them what is best to control and this doesn't match their expectation. I feel that ethnic groups might not see the benefits so easy medications are toxic and to be honest they are but in White patient you might hear oh well at least I tried it but here there might be more thinking going on. [White British, consultant, Male]

Quotes relating to: Moving towards good practice: views on managing future South Asian patients in early inflammatory arthritis clinics

Q25. It is all about rapport building but I don't think I can or ever have achieved with a patient from an ethnic background simply because I have limitations on my part and a fear of

not fully consulting about their health beliefs. So a lost opportunity really. [White British, consultant, Female]

Q26. We are all very busy and in order to move forward we should champion someone for each department who can update every about research in this area and resources around. Like this Apni Jung with NRAS some of know and I met still a lot of people don't know about it. [White British, consultant, Female]

Q27. I think in years to come we do need to understand a lot more during consultation. rheumatology training is all about how to manage the disease but sadly less on how to 
manage the person. So if you could get this onto the rheumatology training for registrars and others then that would be great. [White British, CNS, Female]

Q28. I think that's something we can learn in these training sessions coming from White backgrounds don't always allow the discussions. I am typical middle class person who has little knowledge about different cultures the things I have learnt are what I have picked up

along the way. I think learning more about these models might be more useful in that training for people like me and being verified by people maybe like you who know the research would be a great resource. [White British, consultant, Female]

Q29. We know how to take good medical history but not how to use models in clinic that can

explore patients in this case minority ethnic patients' views about engagement. To feel that confidence in clinic it would be good to know more and more techniques like motivational interviewing skills. I would like to hear South Asians views or heritage they bring to consultation. [White British, consultant, Female]

Q30. We know that there is a need to move forward and do things differently with South Asian patients but fear also if we employed new skills will they work? [White British, CNS, Female]

Q31. I think we should have a programme for professionals as cultures and beliefs are very different and trying to make sense of these can be a job. We only need to look at other specialities like cancer they do... [Afro-Caribbean, registrar, Female]

Q32. A framework to explore illness beliefs, I think someone like yourself who might be used to these concepts can may be use these in clinic but a lot can't do this. So I think something on that will be useful like how to use self-efficacy with patients. [White British, CNS, Female]

Q33. We could be better at this but since we are limited in our full understanding of what really motivates patients or expectations then we are not perhaps getting the whole story or patient on board. We have limited time in the day we should have support from BSR really to do these things. [Afro-Caribbean, consultant, Male]

Q34. The only way we are going to improve this situation if we train and gain more skills on how to manage ethnic minority patients in clinic. I think Arthritis Research UK or BSR should support things like this really. We try our best to follow clinical pathways but something is missing. One though is should clinicians go on compulsory cultural training if you are pointed in a hospital that has so many minority ethnic patients? [White British, consultant, Female] 
Examples of codes

\begin{tabular}{|l|}
\hline Education \\
And different approaches \\
Women with RA tricky \\
Goal setting \\
Leaflets \\
Pictures \\
Pictorial \\
Skills \\
Techniques \\
\hline
\end{tabular}

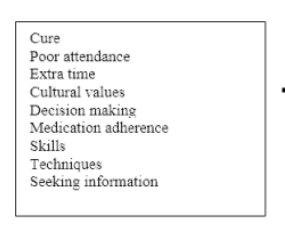

\begin{tabular}{|l|l|l|}
\hline $\begin{array}{l}\text { Grew up in non-ethnic } \\
\text { environment } \\
\text { Rapport } \\
\text { Middle class } \\
\text { White background } \\
\text { Confidence } \\
\text { Skills } \\
\text { Culture awareness } \\
\text { Acculturation }\end{array}$ & $\begin{array}{l}\text { The need for advanced training } \\
\text { of healthcare professional on } \\
\text { dealing with cultural issues }\end{array}$ \\
\hline & & $\begin{array}{l}\text { The need for understanding } \\
\text { theory models in dealing with } \\
\text { cultural issues }\end{array}$ \\
\hline
\end{tabular}

Themes

Varied approaches used in EIA clinic

Experience of managing and delivering information to patients from South Asian background in EIA clinic: rheumatology healthcare professionals' challenges

Engagement with EIA clinic

(doctors nurses and physio)

(a)

Moving towards good practice: views on managing future patients from South Asian background in EIA clinics 